\title{
RESEARCH
}

IN BRIEF

- Intravenous sedation in combination with inhaled nitrous oxide or inhaled nitrous oxide and sevoflurane provides a safe alternative to general anaesthesia in anxious paediatric dental patients.

- With the appropriate staff, training and facilities, these conscious sedation techniques may be safely used in a 'non hospital setting' without the risk of 'deep sedation' or anaesthesia.

- It is essential that future research in the field of paediatric conscious sedation be carried out in the United Kingdom.

\section{An RCT pilot study to test the effects of intravenous midazolam as a conscious sedation technique for anxious children requiring dental treatment - an alternative to general anaesthesia}

\author{
P. A. Averley, ${ }_{1}^{1}$ I. Lane, ${ }^{2}$ J. Sykes, ${ }_{1}^{3}$ N. M. Girdler, ${ }^{4}$ N. Steen ${ }^{5}$ and S. Bond ${ }^{6}$
}

Aim To add to the evidence base for acceptable and effective paediatric conscious sedation techniques in dental primary care.

Objectives To compare three conscious sedation techniques for primary care as an alternative to dental general anaesthesia (DGA) in children. To assess the feasibility and practicality of running the trial in general dental practice. To form the basis for sample size calculations and assess scales of measurement.

Design Single centre, randomised control trial (RCT).

Setting Queensway Anxiety Management Clinic (OAMC). A primary care based general and referral dental practice for the management of anxious patients.

Subjects, materials and methods Sixty five children too anxious for management with relative analgesia, requiring invasive dental procedure for which dental general anaesthesia (DGA) will be required if an alternative cannot

be found.

Interventions Group $1(n=20)-$ A combination of inhaled medical air and titrated intravenous midazolam. Group $2(n=22)-$ A combination of inhaled $40 \%$ nitrous oxide in oxygen and titrated intravenous midazolam. Group $3(n=23)$ - A combination of an inhaled mixture of $0.3 \%$ sevoflurane and $40 \%$ nitrous oxide in oxygen with titrated intravenous midazolam.

Main outcome measures Successful completion of the intended dental treatment with a child who is co-operative and responsive to verbal commands.

Results Fifty per cent (ten children) successfully completed treatment in Group 1, 73\% (16 children) in Group 2 and 83\% (19 children) in Group 3.

1*Principal Dentist, Principal Investigator, 2,3Participating Dentist, Queensway Anxiety Management Clinic, 170 Queensway, Billingham; ${ }^{4}$ Consultant/Senior Lecturer, Sedation Department, School of Dental Science, University of Newcastle upon Tyne: ${ }^{5}$ Statistician, Centre for Health Service Research, University of Newcastle upon Tyne; ${ }^{6} \mathrm{Head}$, School of Population and Health Sciences, University of Newcastle upon Tyne ${ }^{*}$ Correspondence to: Paul Averley, 170 Queensway, Billingham TS23 2NT Email:Paul@averley.com

\section{Refereed Paper}

doi:10.1038/sj.bdj.4811808

Received 11.05.03; Accepted 28.11.03

๑) British Dental Journal 2004; 197: 553-558
This difference was not significant at a 5\% level $\left(\chi^{2}=5.53, \mathrm{df}=2\right.$, $P=0.07)$ Of the total of 20 failures, eight children in Group 1 and one child in Group 2 were successfully treated with the addition of sevoflurane and nitrous oxide in oxygen. Only two children required referral to a hospital setting for DGA and the remaining nine children were managed with an alternative conscious sedation technique. Conclusion This pilot shows that intravenous midazolam especially in combination with the addition of inhaled nitrous oxide or sevoflurane and nitrous oxide were promising safe and effective techniques, sufficient to justify progression to a definitive RCT with appropriate methods.

\section{INTRODUCTION}

This pilot study seeks to investigate intravenous midazolam used in three different conscious sedation techniques. It is hoped these can be used in a primary care setting, (with the appropriate team and facilities) for children requiring dental treatment who would otherwise require referral to a hospital setting for a dental general anaesthesia (DGA).

Child dental anxiety is widespread. ${ }^{1}$ Anxious children can be satisfactorily treated using behaviour management techniques with relative analgesia (RA) if required. Unfortunately for some children, this approach is unsuccessful. ${ }^{2}$ For these children, control of pain and anxiety poses a significant barrier to dental care and DGA is often the only option. Dental treatment under DGA tends to be more radical, with a greater proportion of extractions than fillings. ${ }^{3}$

DGA has been successfully used when RA and behavioural management are ineffective. ${ }^{4}$ However, the risks of DGA have become acutely evident over the past decade. The Department of Health (DoH) in its 'A Conscious Decision' document recognised that although deaths were uncommon during and shortly after DGA (five deaths in dental practices in England in the three years 1996 to 1998 compared with none in the period 1993 to 1995), they were more likely than with any other methods of pain and anxiety management. ${ }^{5}$ Despite their infrequency, deaths associated with DGA are nevertheless difficult to accept, particularly in healthy children. ${ }^{6}$ 


\section{RESEARCH}

Two groups of children pose a particular management problem for dentists:

- Those who are extremely anxious and are unable to cope with treatment with behavioural management or RA.

- Those who require particularly invasive or extensive interventions.

If RA is not appropriate and the risks of DGA unpalatable, is there another option to manage the dental need of these individuals?

\section{Midazolam}

In medical specialities, intravenous (IV) midazolam is gaining popularity as a conscious sedation agent in children.,8 The advantages of IV midazolam in children are: rapid onset of sedation, short duration of action and haemodynamic stability. The safety and tolerability profile of midazolam in children has been described as "comparable or superior to that observed in adults.'

Intravenous midazolam has not been readily accepted as a means of conscious sedation for child dental patient in the UK. There is currently little evidence to support its use. Concerns are twofold: 1) Deeper levels of sedation than intended may be produced; 2) Reaction of children to IV sedation may be unpredictable. ${ }^{9}$ Oral midazolam is, however, gaining popularity and is proving to be both safe and effective. ${ }^{10-12}$

Intravenous midazolam has been successfully used for paediatric sedation in other medical specialities. ${ }^{7,8,13}$ We considered that IV midazolam may be an important additional option for dentistry in providing conscious sedation for children when DGA is considered the only other option.

\section{Sevoflurane}

Sevoflurane is a volatile anaesthetic agent with a sweet, non-pungent odour. It has a low blood-gas coefficient $(0.40) .{ }^{14}$ This allows the depth of sevoflurane inhaled sedation to be carefully controlled when used in sub-anaesthetic concentrations. ${ }^{15}$

The sedative properties of inhaled sevoflurane have been investigated. ${ }^{15-19}$ More recently, the use of inhaled sevoflurane in lower concentrations $(0.1-0.3 \%)$ in addition to $40 \%$ nitrous oxide has been shown to be successful as a paediatric conscious sedation technique with no adverse events. ${ }^{20,21}$ This RCT concluded that the use of sevoflurane in low concentrations (0.1-0.3\%) to supplement nitrous oxide and oxygen for inhalation conscious sedation is safe, practical, and significantly more effective than nitrous oxide alone in children aged 3-10 years having dental treatment. ${ }^{21}$

Queensway Anxiety Management Clinic (QAMC) is part of a large primary care dental practice. QAMC has a professional team of ten dentists, five of whom have their diploma in conscious sedation amongst other postgraduate qualifications, and six part-time consultant anaesthetists who provide full-time cover six days a week. QAMC delivers dental care for more than 4,000 children per year, using a range of conscious sedation techniques. Appropriately trained and experienced dentists administer inhalation sedation with nitrous oxide or if required for children over the age of 16 years, intravenous midazolam. For more anxious children who require more complex techniques, not suitable at present for general practice, operator sedation is not employed. These children are sedated in dedicated facilities with the addition of appropriately trained and experienced consultant anaesthetists, an anaesthetist's assistant and a recovery nurse as part of the team. ${ }^{22}$

Children requiring anxiety management are referred from 190 other dentists in the region. QAMC is one of the first-wave of personal dental service (PDS) pilots, with the specific remit to substitute DGA with conscious sedation. The team has reduced the numbers of referred children requiring a DGA, from 100\% to less than
$10 \%$ over the past 5 years. Over 90\% are now treated using conscious sedation techniques.

Given the large variation in the needs of children, one conscious sedation technique is not enough to manage the needs of all anxious children. With the restriction in availability of DGA services, there is now an urgent need to develop and test a range of conscious sedation techniques for the large number of children who would otherwise require DGA in a 'hospital setting'.

\section{MATERIALS AND METHODS}

This pilot RCT study tests the efficacy of three conscious sedation techniques. Ethical approval and a licence from the Medicines Control Agency was gained. Sixty-five children, ASA I and II, who had been referred to QAMC for dental treatment were recruited. Provided they met the inclusion criteria below:

- Group 1: A combination of inhaled medical air and titrated intravenous midazolam.

- Group 2: A combination of inhaled 40\% nitrous oxide in oxygen and titrated intravenous midazolam.

- Group 3: A combination of an inhaled mixture of 0.3\% sevoflurane and 40\% nitrous oxide in oxygen with titrated intravenous midazolam.

\section{Inclusion criteria}

- Children aged 6-14 years.

- Referred children who, assessed by dentists experienced in the management of anxious children, were unable to accept treatment under local anaesthesia (LA) alone or in combination with RA due to one or more of the following assessments:

1. The child's expressed level of anxiety from a 10-point visual scale $(0-1=$ not scared, $2-3=$ tiny bit scared, $4-5=$ a little more scared, $6-7=$ even more scared, $8-9$ really scared and $10=$ as scared as I can imagine $)^{23}$

2. The dentist's assessment of the child's co-operation using a six point co-operation behavioural scale. $(1=$ relaxed, $2=$ uneasy, $3=$ tense, $4=$ reluctant, $5=$ anxious and $6=$ out of control). ${ }^{24}$

3. The invasiveness of the planned dental procedure using a numerical scale (one point is scored per quadrant of the mouth being treated, plus one point is scored per primary tooth treated, plus two points scored per secondary tooth treated).

- Children are required to have an adequate degree of comprehension and understanding regarding the treatment (if necessary with the support of interpretation services).

- Children are required to accept breathing through a nasal hood and have EMLA cream applied to the dorsum of their hand.

\section{Exclusion criteria}

- Children with hypersensitivity to benzodiazapines, sevoflurane, nitrous oxide or local anaesthetics (all are rare).

\section{The assessment visit}

Professionals involved in the pilot study were formally trained and calibrated to follow the study protocol and its scales. Full verbal and written information about the pilot study was given to the parents of recruited children. Written informed consent was obtained and topical anaesthetic cream (Emla $\left.{ }^{\circledR}\right)$ was supplied. Finally a treatment appointment was arranged.

Children recruited were randomly allocated to one of the three groups following their assessment, using a web-based computer randomisation service.

\section{Sedation technique}

A mixture of lidocaine and prilocaine $\left(\right.$ Emla $\left.^{\circledR}\right)$ cream was applied to the dorsum of both hands of each child by a parent one hour before treatment. 
The child was asked to sit in the dental chair, perform a base line Eve's test (a simple test of spatial awareness in which the child touches the tip of his or her nose with a forefinger with eyes closed) and tolerate breathing through nasal mask. The anaesthetist then opened the envelope inside the record card identifying the technique randomly allocated and commenced its administration.

Group 1: Inhaled medical air at $61 \mathrm{~min}^{-1}$ for 2 minutes. Followed by $0.5 \mathrm{mg}$ of IV midazolam per min. titrated to reach a clinical end point (Level 3 on the consciousness scale).

Group 2: Inhaled 40\% nitrous oxide in oxygen at $61 \mathrm{~min}^{-1}$ for two minutes. Followed by IV midazolam $0.5 \mathrm{mg}$ per min. titrated to reach a clinical end point as described above.

Group 3: Inhaled combination of $0.3 \%$ sevoflurane and $40 \%$ nitrous oxide in oxygen at $61 \mathrm{~min}^{-1}$ for 2 minutes. Followed by $0.5 \mathrm{mg}$ of midazolam per min. titrated to reach a clinical end point as described above.

A Drager Julian anaesthetic machine monitored pulse oximetery, automatic non-invasive blood pressure and ECG. The nasal hood was adapted to incorporate a probe to measure fractional inspired and end-tidal oxygen, carbon dioxide, nitrous oxide and sevoflurane.

Once the clinical end-point was reached, a red toy car was shown to the child for five seconds. The child was asked to recognise the object and memorise it for later.

Topical anaesthetic was then applied to the child's gum. Two minutes later the dentist injected lidocane. During the procedure, the dentist, who was blinded to the technique being used by the anaesthetist, maintained verbal contact and ensured the child remained responsive to verbal commands. The dentist chatted to the child using calming suggestions and imagery, to reassure and to distract attention.

If necessary the concentration of sevoflurane or nitrous oxide was reduced during the procedure if the child showed signs of over sedation (over level 3 on the consciousness scale where, $1=$ awake and not anxious, 2 = awake and anxious, $3=$ partial ptosis and/or slurred speech, $4=$ eyes closed and responds to speech, $5=$ eyes closed, responds to mild physical stimulation, and $6=$ unresponsive to mild stimulation. ${ }^{25}$ Throughout the procedure the established protocols of good sedation practice was employed by the team. ${ }^{5,9,22,26}$

At five-minute intervals, the dentist made a formal assessment of the child's co-operation, using the six-point co-operation scale and level of consciousness. Children were maintained between level 3 and 4 on the consciousness scale.

The anaesthetist continuously monitored oxygen saturation, heart rate, ECG, capnography, fractional inspired sevoflurane and end-tidal sevoflurane and formally recorded them at five-minute intervals during treatment. Blood pressure was recorded once the clinical end point of sedation has been reached.

If a child's level of co-operation rose to level 4 or greater during treatment the technique was deemed to have failed for the purposes of the pilot study. The child received appropriate anxiety management according to the clinic protocols. The nature of the child's anxiety management subsequently employed was recorded.

The intended dental treatment was carried unless limited by the maximum dosage for local anaesthetic. If additional treatment was required a second visit was arranged. This visit was not included in the study.

\section{Recovery}

After treatment, 100\% oxygen was delivered through the nasal hood for two minutes before transfer on a trolley to the recovery room. The child was monitored during recovery by a nurse, who recorded the following:

- Time of the child's arrival in the recovery room.
- Oxygen saturation, heart rate and a blood pressure reading.

- Level of co-operation using the six-point co-operation scale.

- Level of consciousness.

- The time taken for the child to perform an Eve's test tested at five-minute intervals.

- After a completed Eve's test the time taken to walk unaided in a straight line across the room under close supervision.

- Before discharge, the child was asked to recall seeing the toy to assess their level of amnesia.

- The child's level of anxiety and experience of pain 0-1=no hurt, 2-3 = tiny hurt, 4-5 = a little more hurt, 6-7 = hurts even more, $8-9=$ hurts a whole lot more, and $10=$ hurts as much as I can imagine ${ }^{23}$ was reassessed using the visual analogue scales.

- The parent's opinion of the overall management of the child was recorded $(1=$ poor, $2=$ fair, $3=$ satisfactory, $4=$ good, $5=$ excellent).

All the information was recorded on the anxiety management record sheet.

\section{Analytic strategy}

An intention to treat analysis was performed. For each variable considered, initially all three groups were compared simultaneously to test the hypothesis that there were differences between the groups against the null hypothesis that there were no differences. For the key outcome measure (successful completion of dental treatment) and other binary variables a $\chi^{2}$ test was undertaken. For continuous variables a one-way analysis of variance with a standard F-test was undertaken. When the overall test indicated that the differences between groups were significant at the 5\% level, groups were then compared pair-wise. For binary variables a 95\% confidence interval for the relative risk (of success) between groups was calculated; for continuous variables a 95\% confidence interval for the difference in mean scores between the groups was calculated.

\section{RESULTS}

The demographic characteristics of sample, primary and secondary outcomes by group are shown in Table 1 . Fifty-nine children were ASA I and six children were ASA II.

Fifty per cent (10 children) successfully completed treatment in Group 1; 73\% (16 children) in Group 2 and 83\% (19 children) in Group 3. This difference was not significant at a 5\% level $\left(\chi^{2}=5.53 ; \mathrm{df}=2, P=0.07\right)$. Of the total of 20 failures shown in Table 2; eight children in Group 1 and one child in Group 2 were successfully treated with the addition of sevoflurane and nitrous oxide in oxygen. Only two children required referral to a hospital setting for DGA and the remaining nine children were managed with an alternative conscious sedation technique.

There was some imbalance in the groups with respect to gender. By chance there was a much higher proportion of girls in Group 3 than in the other two groups.

For the primary measure of outcome the $\chi^{2}$ test indicated that differences between groups were not significant at the 5\% level $(P=0.07)$. The overall test of significance of differences between groups did not quite reach significance at the 5\% level $(P=0.07)$. Although this was just above the threshold set for further investigation, pair-wise comparisons were carried out to check whether the observed differences between groups were consistent with those used in the sample size calculation for the main trial. These comparisons suggest significantly lower success rates in Group 1 than in the other two groups. The measurements and scales used were reliable between observers and standardised practice was achieved.

There were significant differences between groups in the amount of midazolam required. The pair-wise comparisons indi- 
Table 1 Demographic characteristics of sample, primary and secondary outcomes by group

\begin{tabular}{|c|c|c|c|c|c|c|c|}
\hline \multirow[t]{2}{*}{ Variable } & \multirow{2}{*}{$\begin{array}{l}\text { Group 1: Air } \\
\quad(n=20)\end{array}$} & \multirow{2}{*}{$\begin{array}{c}\text { Group 2: } \\
\text { Nitrous oxide } \\
(n=22)\end{array}$} & \multirow{2}{*}{$\begin{array}{l}\text { Group 3: } \\
\text { Sevoflurane } \\
(n=23)\end{array}$} & \multirow{2}{*}{$\begin{array}{l}\text { Overall test of difference } \\
\text { between groups* }\end{array}$} & \multicolumn{3}{|c|}{ Pair-wise comparison of groups ${ }^{\dagger}$} \\
\hline & & & & & $1 \vee 2$ & $1 \vee 3$ & $2 \vee 3$ \\
\hline Sex (male): $n(\%)$ & $13(65 \%)$ & $15(68 \%)$ & $4(17 \%)$ & $\chi_{2}^{2}=14.5 ; P=0.01$ & RR: $0.95(0.62,1.47)$ & RR: $3.74(1.45,9.64)$ & RR: $3.92(1.54,9.99)$ \\
\hline Age: mean (SD) & $9.3(2.2)$ & $9.6(2.3)$ & $9.9(2.2)$ & $F_{2,62}=0.46 ; P=0.63$ & & & \\
\hline Weight: mean (SD) & $33.6(11.2)$ & $37.6(14.6)$ & $36.1(11.8)$ & $F_{2,62}=0.58 ; P=0.57$ & & & \\
\hline $\begin{array}{l}\text { Invasiveness of } \\
\text { treatment: mean (SD) }\end{array}$ & $9.0(6.3)$ & $9.1(5.8)$ & $10.2(3.7)$ & $F_{2,62}=0.36 ; P=0.70$ & & & \\
\hline
\end{tabular}

Primary outcome

\begin{tabular}{|c|c|c|c|c|c|c|}
\hline $\begin{array}{l}\text { Successful completion } \\
\text { of treatment: } n(\%)\end{array}$ & $10(50 \%)$ & $16(73 \%)$ & 19 (83\%) & $\chi_{2}^{2}=5.53 ; P=0.07$ & RR: $0.69(0.41,1.14)$ & \begin{tabular}{l|l|}
$R R: 0.61(0.38,0.98)$ & $R R: 0.88(0.64,1.21)$
\end{tabular} \\
\hline
\end{tabular}

Secondary outcomes of successful procedures

\begin{tabular}{l|c|c|c|c|c}
\hline $\begin{array}{l}\text { Secondary outcomes } \\
\text { of success }\end{array}$ & $n=10$ & $n=16$ & $n=19$ & \\
\hline $\begin{array}{l}\text { Total dose in mg } \\
\text { of midazolam: mean (SD) }\end{array}$ & $2.9(1.2)$ & $3.5(1.9)$ & $2.5(1.1)$ & $\mathrm{F}_{2,42}=2.3 ; P=0.11$ & \\
\hline $\begin{array}{l}\text { Poorest level of } \\
\text { co-operation during } \\
\text { treatment: mean (SD) }\end{array}$ & $1.9(0.9)$ & $2.0(1.0)$ & $1.9(1.1)$ & $\mathrm{F}_{2,42}=0.03 ; P=0.97$ & \\
\hline $\begin{array}{l}\text { Recovery time in } \\
\text { minutes: mean (SD) }\end{array}$ & $6.5(2.4)$ & $6.9(2.5)$ & $7.6(4.2)$ & $\mathrm{F}_{2,42}=0.44 ; P=0.65$ & \\
\hline $\begin{array}{l}\text { Child's perception } \\
\text { of pain: mean (SD) }\end{array}$ & $1.2(1.3)$ & $0.7(0.9)$ & $1.0(1.5)$ & $\mathrm{F}_{2,42}=0.53 ; P=0.59$ & \\
\hline $\begin{array}{l}\text { Anxiety reported } \\
\text { by child: mean (SD) }\end{array}$ & $1.6(1.4)$ & $1.4(1.3)$ & $2.0(1.9)$ & $\mathrm{F}_{2,42}=0.45 ; P=0.64$ & \\
\hline $\begin{array}{l}\text { Parent's satisfaction } \\
\text { Any recall: } n \text { (\%) }\end{array}$ & $4.6(0.7)$ & $4.8(0.4)$ & $4.4(0.6)$ & $\mathrm{F}_{2,42}=0.21 ; P=0.14$ & \\
\hline
\end{tabular}

Notes: *For binary variables a $\chi^{2}$-test is reported, otherwise an F-test from one-way analysis of variance is reported

${ }^{+} \mathrm{RR}=$ Relative risk [1st group/2nd group] (and 95\% confidence interval) otherwise difference in mean scores (and 95\% confidence intervals) are reported. Pair-wise comparisons are only reported when overall test of difference between groups is significant at the $5 \%$ level.

cate children who received sevoflurane (Group 3) needed less midazolam then children in the other two groups.

Differences between groups in level of parental satisfaction was significant at the 5\% level. Only one of the pair-wise comparisons was significant; parents of children in Group 2 had significantly higher scores than parents of children in Group 3.

Figure 1 and Table 2 show the timing of failure at the technique subsequently employed. Of the ten failures in Group 1, four children failed to co-operate with cannulation and the remaining six during treatment. Of the ten Group 1 failures, eight children successfully completed treatment with the addition of $0.3 \%$ sevoflurane and 40\% nitrous oxide in oxygen. The remaining two children were successfully treated with the addition of a further IV agent ensuring at all times consciousness did not drop below level 4 on the consciousness scale.

of the six failures in Group 2, one child failed to co-operate with cannulation and the remaining five during treatment. Of the six Group 1 failures, one child successfully completed treatment with the addition of 0.3\% sevoflurane and 40\% nitrous oxide in oxygen. Three children were successfully treated with the addition of a further IV agent ensuring at all times consciousness did not drop below level 4 on the consciousness scale. The remaining two children required referral to hospital for DGA.

Of the four failures in Group 3, all children were successfully treated with the addition of a further IV agent ensuring at all times consciousness did not drop below level 4 on the consciousness scale.

No untoward or adverse incidences were encountered during the study.

All children were responsive to verbal command throughout the duration of the procedure and during recovery. (No children scored greater than four on the consciousness scale).

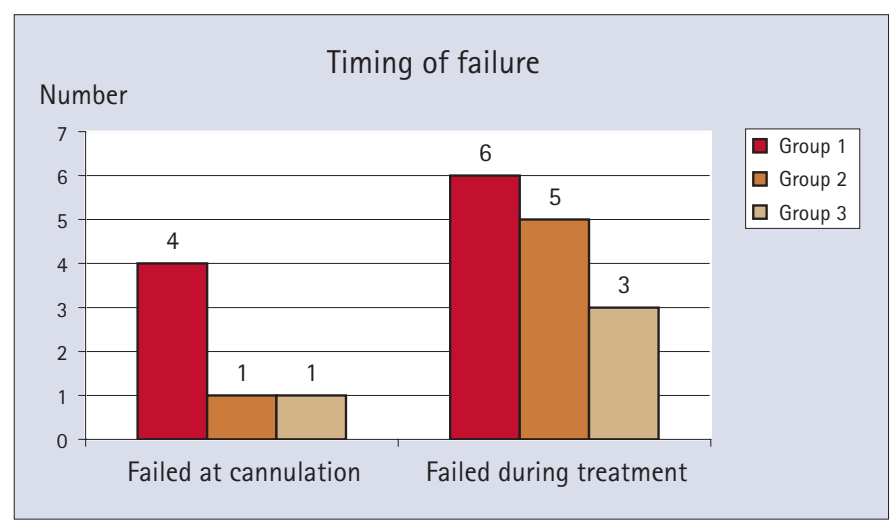

Fig. 1 Timing of failure - the number of children failing to co-operate during cannulation or during treatment

All children remained well saturated and within acceptable limits for conscious sedation during treatment and in recovery. Ninety-eight per cent of children had an oxygen saturation of $98 \%$ or above. The worst recorded saturation of 94\% was one child in Group 1.

Heart rates and blood pressure remained $+/-20 \%$ of normal base values throughout treatment and recovery for every patient.

Table 1 shows Group 3 required less midazolam than Groups 1 and $2(P=0.02)$. The dose of midazolam was not dependent on weight but individually tailored to a clinically titrated endpoint. Differences in recovery times were not statistically significant $(P=0.85)$. There was no statistical significance in child perception of pain $(P=0.39)$ and anxiety in recovery $(P=0.51)$ or parents' satisfaction. 


\begin{tabular}{|c|c|c|c|}
\hline Variable & $\begin{array}{c}\text { Group } 1 \\
\text { IV midazolam } \\
\text { and air }(n=20)\end{array}$ & \begin{tabular}{|c|} 
Group 2 \\
IV midazolam $(n=22)$ \\
and Nitrous oxide
\end{tabular} & $\begin{array}{c}\text { Group } 3 \\
\text { IV midazolam and } \\
\text { sevoflurane }(n=23)\end{array}$ \\
\hline $\begin{array}{l}\text { Addition of } \\
\text { sevoflurane and } \\
\text { nitrous oxide }\end{array}$ & 8 & 1 & 0 \\
\hline $\begin{array}{l}\text { Addition of } \\
\text { other IV agent } \\
\text { (maintaining } \\
\text { consciousness } \\
\text { Level 4) }\end{array}$ & 2 & 3 & 4 \\
\hline $\begin{array}{l}\text { Referral for } \\
\text { general anaesthetic }\end{array}$ & 0 & 2 & 0 \\
\hline $\begin{array}{l}\text { Total number of } \\
\text { failures }\end{array}$ & 10 & 6 & 4 \\
\hline
\end{tabular}

Children in all groups exhibited good amnesia as would be expected with the use of midazolam. Only one child in Group 2 had total recall. Three children in Group 1 and one child in Group 3 had partial recall. Seventeen children in Group 1, 21 children in Group 2 and 22 children in Group 3 had no recall. There was no statistical significance between the groups.

The analysis of secondary outcomes is restricted to subjects who underwent a successful procedure.

\section{DISCUSSION}

Inhalation support (ie a combination of inhalation and intravenous sedative drugs) seems to improve co-operation during cannulation, improves quality of sedation (ie co-operation during procedure), reduces the dose of midazolam required, speeds recovery, offers good amnesia, is safe and effective and reduces referral for DGA. In addition, the inhaled agent can be turned up or down enabling fine control over the depth of sedation if required.

There are two documents for contemporaneous guidance on the use of intravenous sedation for anxious paediatric dental patients. First, 'Maintaining Standards' GDC November 2001 states that: ${ }^{26}$

'In general only one sedative drug (administered by the oral, inhalation or intravenous route) will be necessary in the vast majority of patients. Combinations of sedative drugs may only be justified in exceptional circumstances.'

It is clear from this statement that there is an appropriate concept of minimum intervention in that all attempts should be made to manage childhood anxiety using the standard behavioural management techniques where possible. We believe that children requiring invasive procedures should be supported with appropriate anxiety management. The amnesia that midazolam produces in the event of co-operation failure, is less likely to produce a phobic child. It is likely however, that a significant proportion of anxious children will require some further anxiety management other than relative analgesia alone. In addition, the GDC states that:

'Intravenous conscious sedation can rarely be justified in children.'

Intravenous conscious sedation in children can be justified, to avoid general anaesthesia and when other treatment options have been explored.

The second reference document in the United Kingdom for guidance has been produced by the Scottish Intercollegiate Guidelines Network (SIGN) and is titled 'Safe Sedation of Children Undergoing Diagnostic and Therapeutic Procedures, 2002'.2 This document is the most extensively researched and comprehensive review of sedation techniques for treatment in medical and dental patients undergoing hospital or primary care procedures. The SIGN document has a dedicated chapter for sedation requirements in relation to dentistry. It states that:

'Intravenous sedation is seldom indicated for children under the age of 16 years. Single agent sedation with midazolam is only recommended for intravenous dental sedation in patients over 16 years of age. Intravenous sedation should be avoided in younger children in primary or community dental practice.'

However, the evidence put forward for the above statement is weak and not been relevant to dentistry or is from the USA where 'deep sedation' is practised. The extrapolation of the side effects of these techniques for conscious sedation may not be dose dependent or evidence has been ranked as grade 4 (scale $1=$ high quality meta-analysis, scale $4=$ expert opinion with no sound evidence base to support statement).

It is essential that future research in the field of paediatric conscious sedation be carried out in the United Kingdom. This research should be high quality and co-ordinated in developing the evidence base for paediatric conscious sedation. If children are unable to co-operate with the support of RA it is imperative that they are able to access care without resorting to DGA. Current guidance could increase referrals for DGA placing further pressure on these services and not working in the best interest of patients. A range of evidence-based conscious sedation techniques need to be established so that the formulation of guidance is well informed and appropriate.

Safety is paramount for any conscious sedation technique. It is widely accepted that conscious sedation is safer than general anaesthetic. ${ }^{2,25,27-30}$ However, a poorly controlled conscious sedation technique may result in deep sedation or general anaesthesia with all its attendant risks. ${ }^{2,31}$ Unintended loss of consciousness may be potentially more risky than general anaesthesia. ${ }^{2}$ The sedationist must be able to exert a fine control over level of sedation. The margin of safety between sedation and anaesthesia must be wide enough to prevent unintended loss of consciousness occurring. The current gold standard of postgraduate training for conscious sedation is the Diploma in Conscious Sedation. This equips dentists well to deliver RA to children and intravenous midazolam to adults. Children requiring more complex techniques involving combinations of drugs for effective sedation should be treated in specialist centres with appropriately trained and experienced teams. It is appropriate that these centres operate in a 'non hospital setting'.

On the basis of these encouraging findings we have been able to test our study protocol. In addition we have been able to make power calculations for the main RCT and develop a definitive RCT comparing the three interventions described above. The main study is now completed. It is anticipated that results will be available by the winter 2003 .

The authors thank all the patients involved in this pilot study. They are grateful for the support of an NHS R\&D National Primary Care Researcher Developers Award (2002). They thank consultant anaesthetists; I. Riddle, G. Lahoud, S. Gooneratne, $N$. Syresh and $H$. Mohan for their participation in running the studies. In addition we thank dental surgeons, S. Brown, M. Hanlon, O Hobman, U. Mansoor, B. Smith and A. Weston for their valuable contribution. We thank all the staff at QAMC for their hard work and Abbott Laboratories for providing the sevoflurane.

1. Veerkamp J S, Gruythuysen R J, van Amerongen W E, Hoogstraten J. Dental treatment of fearful children using nitrous oxide. Part 2: The parent's point of view. ASDC J Dent Child 1992: 59: 115-119.

2. Scottish Intercollegiate Guidelines Network. Safe sedation of children undergoing diagnostic and therapeutic procedures. A national clinical guideline. 2002.

3. Harrison M, Nutting L. Repeat general anaesthesia for paediatric dentistry. Br Dent J 2000; 189: 37-39

4. Holt R D, Chidiac R H, Rule D C. Dental treatment for children under general anaesthesia in day care facilities at a London dental hospital. Br Dent J 1991; 170: 262-266. 
5. Department of health. A conscious decision. A review of the use of general anaesthesia and sedation in primary dental care. London: Department of Health 2000.

6. Worthington L M, Flynn P J, Strunin L. Death in the dental chair: an avoidable catastrophe? Br JAnaesth 1998; 80: 131-132.

7. Rosen D A, Rosen K R. Intravenous conscious sedation with midazolam in paediatric patients. Int J Clin Pract 1998; 52: 46-50.

8. Shannon M, Albers G, Burkhart K, Liebelt E, Kelley M, McCubbin M M et al. Safety and efficacy of flumazenil in the reversal of benzodiazepine-induced conscious sedation. The Flumazenil Pediatric Study Group. J Pediatr 1997; 131: 582-586.

9. Hosey M T. UK National Clinical Guidelines in Paediatric Dentistry. Int J Paediatr Dent 2002; 12: 359-372.

10. Erlandsson A L, Backman B, Stenstrom A, Stecksen-Blicks C. Conscious sedation by oral administration of midazolam in paediatric dental treatment. Swed Dent J2001; 25: 97-104.

11. Wilson KE, Welbury R R, Girdler N M. A randomised, controlled, crossover trial of ora midazolam and nitrous oxide for paediatric dental sedation. Anaesthesia 2002; 57: 860-867.

12. Wilson KE, Welbury R R, Girdler N M. A study of the effectiveness of oral midazolam sedation for orthodontic extraction of permanent teeth in children: a prospective, randomised, controlled, crossover trial. Br Dent J 2002; 192: 457-462.

13. Alcaino $E A$. Conscious sedation in paediatric dentistry: current philosophies and techniques. Ann R Australas Coll Dent Surg 2000; 15: 206-210.

14. Rang H P, Dale M M, Riller S H. Pharmacology. 4th edn, 1994.

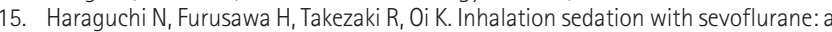
comparative study with nitrous oxide. J Oral Maxillofac Surg 1995; 53: 24-26.

16. Ganzberg S, Weaver J, Beck F M, McCaffrey G. Use of sevoflurane inhalation sedation for outpatient third molar surgery. Anesth Prog 1999; 46: 21-29.

17. Hoerauf $\mathrm{K} \mathrm{H}$, Hartmann T, Zavrski A, et al. Occupational exposure to sevoflurane during sedation of adult patients. Int Arch Occup Environ Health 1999; 72: 174-177.
18. Ibrahim A E, Taraday J K, Kharasch E D. Bispectral index monitoring during sedation with sevoflurane, midazolam, and propofol. Anesthesio/2001; 95: 1151-1159.

19. Katoh $\mathrm{T}$, Bito $\mathrm{H}$, Sato $\mathrm{S}$. Influence of age on hypnotic requirement, bispectral index, and 95\% spectral edge frequency associated with sedation induced by sevoflurane. Anesthesio/2000; 92: 55-61.

20. Lahoud G Y, Averley PA, Hanlon M R. Sevoflurane inhalation conscious sedation for children having dental treatment. Anaesthesia 2001; 56: 476-480.

21. Lahoud G Y, Averley PA. Comparison of sevoflurane and nitrous oxide mixture with nitrous oxide alone for inhalation conscious sedation in children having dental treatment: a randomised controlled trial. Anaesthesia 2002; 57: 446-450.

22. Averley P A. Queensway Anxiety Management Clinic Referral Protocols. www.anxietymanagement.co.uk. 2003.

23. Wong D L, Baker C M. Children's visual and verbal rating scale. Paediatr Nurs 1988 1988; 14: 1.

24. Venham L, Quatrocelli $S$. The young child's response to repeated dental procedures. J Dent Res 1977; 56: 734-738.

25. Girdler N M, Hill CM. Sedation in dentistry. 1st edn. London: Wright publications, 1998

26. General Dental Council. Maintaining Standards November 2001. London: GDC, 2001.

27. Clinical guideline on the elective use of conscious sedation, deep sedation and general anaesthesia in pediatric dental patients. Chicago: American Academy of Pediatric Dentistry (AAPD), 1998

28. Department of Health Review Group Chaired by the Chief Medical and Dental Officers. A Conscious Decision. London: Department of Health, 2000.

29. Department of Health. Department of Health circular (from the director of Health Services), letter to all GDPs, Health Authorities, NHS trusts and Dental Practice Board. London: Department of Health, 2001.

30. Malamed S F. Sedation, a guide to patient management. 3rd edn. Boston: Mosby publications, 1995.

31. Morton N S, Oomen G J. Development of a selection and monitoring protocol for safe sedation of children. Paediatr Anaesth 1998; 8: 65-68. 